\title{
Arbor
}

\section{Redentores irredentos. Los diarios madrileños después del 98}

\section{Jesús Timoteo Alvarez}

Arbor CLXIX, 666 (Junio 2001), 541-556 pp.

\section{La consolidación de un nuevo sector industrial}

La historia del periodismo en el periodo de la Restauración está oficialmente muy acabada. Probablemente es aún —en competencia ahora con los años de la guerra civil- el periodo más conocido y con una interpretación consolidada en la que están de acuerdo la mayor parte de los historiadores (Sánchez Aranda/Barrera, 1992; Fuentes/Fdz Sebastían, 1997).

La novedad más importante del cambio de siglo es la consolidación de un nuevo sector industrial, el de la prensa. Desde muchos puntos de vista, de los que a continuación citaremos los más importantes, los periódicos eran un valor minoritario y secundario antes de 1875 , dentro de una sociedad y un sistema en el que apenas tenían interés (Timoteo,1981), mientras que en torno a 1900 nos encontramos ya con un sector industrial desarrollado y consolidado, con agentes conocidos que, los más notables, han atravesado el siglo XX español como sagas industriales definidas: nos referimos a La Vanguardia y los Godó y al $A B C$ y los Luca de Tena, pero también a los Gasset-Ortega con El Imparcial-El Sol-El País y a diarios cabeceras de región como El Heraldo, El Correo Español, Las Provincias y el Mercantil Valenciano-Levante, La Voz, El Correo de Andalucía, etc.

Los nervios sobre los que ese sistema industrial se consolida son, básicamente, los que siguen. En primer lugar el establecimiento de un régimen legal definido en torno a la ley de 1883. Con todos los inconvenientes conocidos, la ley, que formalmente se mantendrá hasta la segunda República, constituye el marco de referencia en torno al 
cual se desarrolla la nueva prensa, la que Mainer definía como «empresa» en contraposición a la prensa de partido, y que coincidía con la generalización en las primeras ciudades del mundo de la primera oleada del «nuevo periodismo» o periodismo de masas.

En segundo lugar la industrialización de las materias primas, la producción, la distribución y las ventas: la modernización de la imprenta con la composición mecánica de textos y la producción masiva de ejemplares, la producción industrial de papel y de tinta, la posibilidad de una distribución relativamente rápida a través de la red de ferrocarriles ya terminada en toda Europa, la operatividad también terminada de la red mundial de telégrafos y correos, el establecimiento de fórmulas de venta alternativas (subscripciones y venta callejera).

En tercer lugar la transformación del producto. El periódico aumenta el número de páginas, diversifica los contenidos y los organiza en secciones, se especializa, incluye el diseño con aires de cartel en la portada y contraportada sobre todo e incluye fotografías, aligera el texto, define titulares y ladillos, populariza el estilo, incluye viñetas, incluye servicios e informaciones de interés general, define una política de captación y precios de publicidad, etc.

En cuarto lugar la profesionalización de los empresarios y de los periodistas. En torno a 1900 o poco después nacen las escuelas de periodismo, comienzan a aparecer y a conocerse balances y cuenta de resultados de los periódicos y se clarifica una mentalidad de negocio en la que las fuentes abiertas de ingresos (ventas y publicidad) queden claramente marcadas. Es lógico así que los grandes diarios arrastren la aparición de sectores industriales afines y dependientes como la publicidad, la imprenta, la distribución y sus componentes (hoy logística) (Gómez Mompart, 1989).

En quinto lugar -aunque en lógica clásica sea la primera- la evolución general, social y económica, de España: el trasvase de población del campo a las ciudades, la industrialización, la generalización de la enseñanza, el crecimiento en todos sus ámbitos. En estadísticas recientemente publicadas, Julio Alcaide (2000) ( La renta nacional de España y su distribución. Serie años 1898 a 1998») (J.Velarde Fuertes, 1900-2000.Historia de un esfuerzo colectivo. Fundación BSCH-Planeta, 2000) presenta una tasa del 2,24 por ciento de crecimiento acumulativo anual entre 1898 y 1935, tasa -aunque inferior al $4 \%$ de media para el periodo 1935-200 - que, como media anual, es considerada como impresionante y muy difícil de mantener en opinión de Velarde.

En realidad estaba teniendo lugar una radical transformación en las cabezas de los ciudadanos occidentales (en torno a 1900) que iba 
a definir, por encima probablemente de cualquier otra consideración política y económica, al individuo del siglo XX, encuadrado en una sociedad de masas. Me refiero, por una parte, a la aparición de un «hombre nuevo», («no sabemos que nos pasa: eso es lo que nos pasa» de Ortega), el «hombre sin atributos» $\mathrm{y}$ «sin fundamentos» que ha perdido las referencias axiológicas de una sociedad agraria tradicional y burguesa tradicional (Timoteo, 1987) y, por otra parte, al nacimiento de una mentalidad de compra-consumo de bienes y servicios como exigencia vital prioritaria, como definidora de estatus y situación social, como elemento identificador del individuo y como demostración de vitalidad, supervivencia, personalidad y estilo, vida. La ansiedad individual transformada en exigencia colectiva y la necesidad de consumir definen el siglo XX. Esas necesidades forzaron la reorganización de la estructura social entera, de los partidos políticos y de los Parlamentos, de toda la producción y el comercio, a la invención del marketing y de las cadenas y de las grandes superficies, y de la popularización de la banca, etc., y obliga al desarrollo de la industria de la publicidad y a la popularización de los medios de información y comunicación, que naturalmente se convierten poco a poco en rentables, en importantes negocios.

Este nuevo sector industrial nace en todo occidente y también en España. El resultado es que, según las Estadísticas oficiales de la prensa periódica, teníamos 1.347 publicaciones periódicas en España en $1.900,1.980$ en 1.913 y 2.289 en 1.920 .

Los exponentes más llamativos y conocidos de esa consolidación industrial de la prensa o del sector de los medios de comunicación son: el «trust» de El Liberal, La Vanguardia, el $A B C$, la Editorial Católica y $E l$ Sol. Cada uno de estas sociedades y medios están suficientemente analizados. Todos ellos supusieron, en una instancia evidente, estrategias empresariales que implicaron inversiones y capitalización, equipos profesionales, perspectivas a largo plazo, objetivos de negocio, etc. El Liberal contaba en 1901 con una economía suficientemente saneada como para crear cuatro diarios con el mismo título en Barcelona, Bilbao, Sevilla y Murcia, éste último como transformación de Las Provincias, comprar también El Defensor de Granada y El Noroeste (Gijón) y sobre todo constituir en 1.906 la «Sociedad Editorial» conocida como «el trust» y a la que se incorporan El Imparcial y El Heraldo. La Vanguardia, nacida años atrás (1881) como diario vinculado al Partido Liberal y movido después a posiciones conservadoras, se consolida como empresa desde 1897 con la llegada a la dirección de Ramón Godó y domina el mercado barcelonés desde 1.903. Para 
1920 era un saneado negocio y uno de los primeros diarios españoles. $E l A B C$, cuya empresa arranca en 1891 con la creación de la revista Blanco y Negro (incorporó novedades importantes a la edición como el fotograbado, el color y la foto en color), nace en 1903 instalado ya en un amplio y notable edificio en la calle Serrano y se confirma en 1909 con la creación de la sociedad Prensa Española (tres millones de capital inicial de la época). En 1915 reconoce ingresos por publicidad de 800.000pts, una tirada diaria de 73.000 ejemplares y beneficios saneados. La prensa católica, siguiendo las recomendaciones de León XIII, arranca con El Correo de Andalucía (1899), continúa con La Gaceta del Norte (Bilbao) en 1901 y culmina con la aparición de El Debate (Madrid, 1910) y de La Editorial Católica (1912). En torno a 1918 se le estima a El Debate una tirada diaria de 40/45.000 ejemplares y una notable influencia en la sociedad española. El Sol arranca en 1917 con la idea clara de crear un gran grupo de comunicación desde el principio: precedido por la experiencia de Urgoiti hijo en Estados Unidos, Nicolas de Urgoiti pone en marcha dos diarios ( $E l$ Sol y La Voz), una revista (Voluntad), una agencia de noticias (Febus), una editorial (Calpe-Espasa), una agencia de publicidad (Urgoiti,Salas y Porrero) y todo ello con el apoyo de Papelera Española y con sociedades vinculadas (Prensa Gráfica, editora de Mundo Gráfico, Nuevo Mundo y La Esfera, ofrecidas en suscripción combinada con El Sol). Es evidente que pensaba en un poderoso grupo económico.

En una primera impresión parece, pues, que, a la altura de 1920, existía en España, Madrid sobre todo y Barcelona, un sector informativo industrializado y consolidado con, al menos, los citados cinco grupos editores en plena actividad y resultados.

La situación, sin embargo, era más compleja porque estos grupos constituidos eran y estaban obligados a ser más que empresas o tal vez menos que empresas, en cualquier modo algo distinto. Ejercieron conscientemente un papel de redentores de la sociedad española, con un alto precio empresarial para quienes se empeñaron de lleno en la función.

\section{El gen misionero o la función salvífica de la prensa española de 1900}

La sociedad española vivió con una generalizada sensación de vacío histórico y fracaso los años que van de 1897 (asesinato de Cánovas) a 1902 (mayoría de edad de Alfonso XIII) con el desastre colonial de 
1898 como dominante (Seco, 1993). Esa frustración se tradujo en una profunda crisis de confianza como prueba irrefutable de la decadencia nacional: mientras el resto de las metrópolis ampliaban su poderío colonial, España perdía sus últimos territorios en Cuba, Puerto Rico y Filipinas.

La frustración consecuente con la derrota de 1898 se popularizó gracias sobre todo al entusiasmo informador y provocador de los diarios, quienes, emulando a los grandes diarios sensacionalistas de Nueva York, implicaron a los españoles en la contienda. Igual que en Nueva York, en Madrid se intentaban vender más diarios (el caso de $E l$ Liberal y sus cifras es el más conocido) y, para ello, imitando a los americanos, se intentó llevar a cabo aquí un primer ensayo de propaganda masiva organizada (Timoteo, 1987).

Incluso el movimiento literario del 98, inevitablemente unido a los conceptos de decadencia y pesimismo, nace y se desarrolla en torno a Los Lunes de El Imparcial. «La generación del 98 es fundamentalmente una operación de marketing, un movimiento social creado desde y por los periódicos y un claro ejemplo de la función de agentes sociales que los medios de comunicación adquieren en el siglo XX" (Timoteo, 1989). Según recogen Villacorta (1980) primero y Carmen Cafarell, después, en su no publicada tesis sobre Ortega Munilla, director de Los Lunes entre 1879 y 1906, en este suplemento se «lanzaron» Unamuno (20 de mayo de 1895), Benavente (3 de junio de 1895), Azorín (que publicó aquí La ruta de Don Quijote), Pio Baroja, Valle Inclán y Ortega y Gasset (14 de marzo de 1904) (Mainer, 1975).

Esa concepción generalizada de que la prensa podía alcanzar extraordinarias influencias sobre las mentes y los comportamientos de las masas terminó por hipnotizar a los agentes (periodistas, directores, empresarios y políticos) del sector y por reorientar los fines y objetivos, racionalmente empresariales, hacia otros objetivos más políticos y con toda seguridad menos rentables a corto plazo.

Sucedió en todo Occidente pero en España esta capacidad de los periódicos para crear conciencia fue tan generalizada y temida que marcó de modo decisivo las primeras décadas del siglo XX. «Los curas campesinos maldicen desde el altar a todos los suscriptores de los periódicos rotativos. La revolución se considera aquí como una vasta difusión de Imparciales». La cita de Julio Camba en 1916 recoge esa consciencia, como la recogen Cansinos-Assens o Corpus Barga o como Anton del Olmet presume de haber creado la figura de Maura: "Así pinté yo a Maura. Al cabo de escasos dos años de acción, realizada desde el primer diario español, con el prestigio que yo tenía entonces, media España era maurista». 
En la conciencia popular de entonces, los periódicos eran responsables de lo bueno y de lo malo, del 98 para unos, de la capacidad de recuperación del espíritu para otros, eran en definitiva los primeros instrumentos de una hipotética educación popular, imprescindibles en una hipotética regeneración de la sociedad y de la política española. $\mathrm{Y}$ como los movimientos más llamativos del fin de siglo, abocados al pesimismo y al decadentismo y por tanto al regeneracionismo, coincidían con objetivos de fuerzas sociales emergentes (socialismo, anarquismo) en la necesidad de reconquistar las mentes y de forjar hombres nuevos, el resultado parece inevitable.

Prácticamente toda España se vuelve misionera, empeñada en recuperar para sus propios ideales y fines a todos los demás y absolutamente seguros de que el periódico propio es el instrumento decisivo en esa conquista. Apoyando movimientos educadores, por supuesto.

La salvación de España es una cuestión pedagógica, un problema pedagógico y la pedagogía social es el primer problema política (slogan de Ortega en su famosa conferencia del 12 de mayo de 1910). Regenerar: Costa, Ganivet y Maeztu, Primo de Rivera, la segunda República, Polavieja, Silvela, Maura, Canalejas, Alba, militares, colegios profesionales, institucionistas, católicos, anarquistas, socialistas, intelectuales del 98 . y del 14 en Madrid, Barcelona, Comillas, Deusto, El Escorial, etc., todos intentaron transformar a la sociedad española y a las masas por medio de la educación política y la propaganda, es decir y sobre todo, por medio de los periódicos.

«En España la cultura está muy atrasada, sobre todo, está muy poco extendida. Son poquísimos los lectores de libros. El periódico empieza a leerse bastante. Los obreros, los humildes, buscan con avidez el impreso barato...y alimentan su espíritu con lo que les dan.En España empieza a haber ahora una gran tribuna para la enseñanzà popular y no se aprovecha: el periódico, tanto como el maestro, que pone el medio, el saber leer, importa el periodista, que pone el fin, lo que el pueblo debe leer (Clarín, «Los periódicos»: El Español, 28/1011899). «Ante los enormes estragos de la prensa anticristiana urge darse cuenta de la gran catástrofe que nos amenaza y disponerse para la defensa.Urge levantar la prensa católica a la altura que le corresponde, tal, que infunda respeto a nuestros propios adversarios. Urge organizar una cruzada de la Buena Prensa, si es que no queremos que la nación de Pelayo y San Fernando, la nación de la Inmaculada, la nación católica, sufra el terrible castigo de que el sol de la fe se eclipse de su purísimo cielo» («Asamblea Nacional de la Buena Prensa» edit. El Correo de Andalucía, Sevilla, 1905). Son dos testimonios entre los muy 
abundantes que pueden recogerse de esa generalizada consciencia y de esa época, la de una pasión redentora no fácil de entender en nuestros días.

Es en ese ámbito donde se desarrollan las nuevas empresas periodísticas. Con mayor o menor implicación, los grupos informativos industriales y específicamente los cinco grupos a que más arriba hemos hecho referencia, se implicaron de lleno en la regeneración de España. Específicamente y sobre todo la Editorial Católica con $E l$ Debate y el grupo de Urgoiti con $\mathrm{El} \mathrm{Sol}$ como cabecera líder. Ambos grupos jugaron muy fuerte, implicándose de tal manera que su bien organizada estructura fue devorada por los avatares de la historia política inmediata.

Junto a esos grupos grandes, conocidos en la época como «prensa industrial», mantenían el afán misionero todo tipo de hojas periódicas de organizaciones socialistas y anarquistas, sí, pero también de organizaciones cristianas, de librepensadores, de naturistas, de sectores profesionales. España era, a la altura de 1900-1914 una inmensa tierra de misión donde todos estaban empeñados en regenerar al resto, a todos los demás.

\section{Los debates sobre el origen de la cultura y de la prensa de masas en España}

La sociedad española vivió el principio de siglo con una dolorosa sensación de frustración y fracaso, por una parte, y con una imperiosa necesidad de regeneración y espíritu misionero, por otra.

La situación real posterior al desastre no era, sin embargo, tan negra como fue percibida por los coetáneos. No sólo las citadas cifras de Alcaide lo vienen a demostrar ahora sino otros muchos indicadores económicos favorables han sido puestos de manifiesto por los historiadores (Salaün/Serrano: 1991).

¿Por qué entonces el enorme pesimismo de todos los noventayochistas? ¿por qué el éxito popular de los alegatos de Costa sobre «Si España posee aptitudes para ser una nación moderna» (1908) o del «manifiesto» de Silvela «Sin pulso» (1898: 16 de agosto en El Tiempo)?

Las tesis de Costa y de la generación del 98 se basaban en algunas cifras reales (parcialmente utilizadas y amplificadas): es cierto un porcentaje de analfabetos alto en España para 1900 (63\%), superior a la media de Europa occidental (50\%) y superior sobre todo al poco creíble de Francia (16\%), cifras que en 1918 se reducían ya al 50\% de media para España según datos de Luzuriaga recogidos por $E l$ 
Sol, como es exacta la comparación entre grandes metrópolis en onda expansiva en un mundo colonial y España en retroceso. A partir de esos y similares datos (niveles de población urbana, niveles de industrialización, etc.) todos los seguidores del pesimismo histórico han culpado a la burguesía española de no ser capaz de moverse al mismo ritmo que la europea en el desarrollo industrial, dominada por el interés individual y mezquino e incapaz de poner en pie un régimen político solvente. Una buena parte de los escritores del 98 amplían esa responsabilidad a toda la sociedad española carente de pulso colectivo.

Los analistas sobre la prensa en el cambio de siglo se han encontrado desde el primer momento con esa situación: ¿eran los diarios españoles realmente retrasados respecto a los coetáneos del resto de Europa? ¿estaba la razón en que no existía en España una sociedad de masas y por tanto no era posible la existencia de diarios de masas? ¿era culpa de la burguesía española, incapaz de adecuarse al ritmo de los tiempos y de generar grupos empresariales acordes con los de París o Londres o era culpa de la sociedad española visceralmente atrasada y sin interés en adecuarse a los tiempos nuevos?

El debate se vivió con intensidad entre los expertos a lo largo de los 80: Desvois, Almuiña, Seoane, Timoteo, Moreno Sardá, Gomez Mompart, Treserras, Guereña, etc. (Gomez Mompart, 1989) y la conclusión parece, en cuanto a nuestro país se refiere, bastante aceptada: cuantitativamente hablando (por tirada e ingresos), los grandes diarios de Madrid no alcanzaron las cifras de los grandes diarios de París, Nueva York o Londres, pero cualitativamente hablando se dieron en España periódicos diarios con muchas de las cualidades y, sobre todo, objetivos y funciones de los medios de masas.

Esta conclusión, sin embargo, es exclusivamente técnica y no resuelve o se salta el dilema sobre reparto de culpabilidades (¿la burguesia incapaz o la sociedad que se niega a evolucionar?) y traslada el debate teórico a otros ámbitos: tal vez no exista culpabilidad sino complejo o tal vez los análisis sobre la realidad española han estado demasiado influidos por el espíritu autista característico de sociedades atormentadas.

Por eso la polémica ha iniciado un salto importante, que considero ausente de complejos y con gran futuro. Por ejemplo, Jaume Guillamet, catedrático de Historia de la Comunicación en la Universidad Pompeu Fabra de Barcelona, define en su Memoria de Cátedra (2000) «Factores de Progreso y Atraso» como metodología básica para el análisis de la historia del periodismo español y catalán. $\mathrm{O}$, por ejemplo y en el ámbito más general de la teoría de la Comunicación, se están moviendo los 
investigadores en torno a la adecuación de los medios a las diversidad de las sociedades, es decir, en torno al problema de si los medios son algo más que productos de mercado, agentes con funciones específicas en las sociedades del siglo XX.

La propuesta de Guillamet es muy interesante. Arranca de la puesta en duda del principio anglosajón de que el periodismo es un invento americano y plantea un análisis comparado de las principales tradiciones periodísticas «en base a la interacción de un conjunto de factores de progreso y de atraso, como vía de explicación de los distintos elementos de estabilidad, continuidad, alteración, aceleración o desactivación de los procesos evolutivos». Guillamet cita antecedentes atractivos de su propuesta ( CHALABY,J.K., «Journalism as an anglo-american invention" en European Journal of Communication, XI, 3, 1996; FERENCZI, T., L'invention du journalisme en France. Naissance de la presse moderne a la fin du XIX siècle, Olon, Paris, 1993; PIZARRoso, A., Historia de la Prensa, C. Ramón Areces, Madrid, 1994) pero presenta un proyecto bastante definitivo con una caracterización de las principales tradiciones periodísticas de las que extraer una indicación de factores definitorios de progreso y atraso. Se refiere a Italia, Alemania, Francia, Inglaterra, España y Estados Unidos y enumera como líneas de análisis las siguientes:

Factores de progreso:

1. Actuación de los gobiernos de acuerdo con el espíritu de las épocas y tipos de régimen.

2. Ritmos avanzados de instauración del liberalismo político y económico.

3. Procesos de crisis y revolución política.

4. Desarrollo económico y social.

5. Relación industria-medios de comunicación.

6. Posiciones de influencia internacional, imperial y colonial.

7. Estructuras políticas territorialmente próximas o descentralizadas.

8. No Intervención de los gobiernos en los modelos audiovisuales.

Factores de atraso:

1. Actuación de los gobiernos contraria al espíritu de la época y tipos de régimen.

2. Obstáculos a la instauración đel liberalismo político y económico y al desarrollo económico y cultural.

3. Efecto directo de las guerras y de los regímenes totalitarios. 
El esquema nos lleva a una investigación comparada de singular interés para el conocimiento de la historia del periodismo y de la comunicación en España, excesivamente autista o sometida a la comparación con sistemas informativos más próximos (el francés) o dominantes (el norteamericano), sin una posición propia en el ámbito occidental en su conjunto. Así por ejemplo, dice Guillamet, «la influencia en la formación de las tradiciones periodísticas de las antiguas colonias de América sería (para España) un factor de progreso que no se da con el mismo alcance en Francia ni mucho menos en Italia o en Alemania». Espero con mucho interés los resultados de este trabajo, que, dada la capacidad de Guillamet, no tardaremos en tener disponibles.

En un ámbito paralelo conviene considerar las aportaciones metodológicas que la postmodernidad aporta a nuestro campo de actividad, el de la Historia de la Comunicación. José Luis Pinillos concluye su detallado análisis de los pensadores y del pensamiento postmoderno (En el corazón del laberinto. Espasa. Madrid, 1998) con una muy adecuada sentencia: «...el hecho es que no hay ya teorías unitarias, como en su tiempo fueron la mecánica de Newton o el evolucionismo de Darwin, capaces de dar razón a la complejidad del mundo en que vivimos.. El problema estriba en que, a pesar de todo, sigue siendo necesario encontrar un cierto equilibrio entre la libertad y el orden. Mal que bien las democracias liberales habían resuelto a su modo la cuestión, hasta que el advenimiento de la sociedad de masas, la descolonización, las nuevas tecnologías y otros factorés por el estilo acabaron por desequilibrar la situación... Ahora...ha surgido una cultura postmoderna que defiende tenazmente el pluralismo porque se halla persuadida de que el ser y la experiencia son ambiguos, se dicen de muchas maneras... Al mundo moderno le ocurre que son dos las almas que hay en él... Una es la razón encargada de que cada individuo ocupe su lugar exacto en el sistema. La otra no es sino un corazón que aspira a latir en libertad. Si hay algo que se haya puesto de relieve en el debate de la postmodernidad es que un alma ha crecido a expensas de la otra y que la humanidad necesita de las dos».

Traducir estos principios a la metodología de la Historia de la Comunicación significa jugar con factores contradictorios: ¿es posible el análisis con resultados de procesos complejos e inabarcables? ¿es posible comparar realidades sociales solo analógicas y procesos de comunicación que no se identifican -en contra de las historias nacionales dominantes hasta hace nada - con países sino con ciudades y ámbitos de influencia de las metrópolis (no hubo medios de masas en Oregon sino en Nueva York ni en Tours sino en París)? (Gómez Mompart, J.: 1996). 


\section{Redentores irredentos. Los diarios madrileños}

Vamos a intentar resumir a continuación los componentes de este debate. Opino que son propuestas de trabajo valientes y que recogen muy bien los campos en que las teorías analíticas de la comunicación deben, en mi opinión, moverse.

1. La comunicación define lo propio y específico, vertebra los grupos, integra y sistematiza lo complejo.

El axioma, que es casi universal desde Habermas, se puede hacer postmoderno a partir de una cierta teoría de redes, muy querida en nuestros ámbitos por Moreno Sardá (1998) y según la cual el mundo de la comunicación carece en nuestros días de centros de referencia como lo fueron en su momento París, Londres o Nueva York, a medida que el peso en el Cartel de Agencias se desplazaba de una a otra de las citadas ciudades. Cada uno, individualmente, cada medio, cada grupo, cada sistema, arrancan el día como centro de una red mundial ( y no sólo a través de la www) desde su propio territorio específico, con capacidad para explícitamente aceptar o rechazar valores externos y con capacidad para promocionar los propios.

El juego se define así en un campo de poder diluido, donde cada agente está obligado a buscar alianzas (consenso) con otros agentes para que sus posiciones tengan posibilidades de ser conocidas, primero, y atendidas o rechazadas. Es evidente que la capacidad y el poder de los agentes es muy dispar: no es lo mismo el peso específico en ese espacio de consenso y de poder diluido de un Estado o de una Corporación mundial que el de un grupo de presión local o de una asociación de ecologistas, pero es igualmente evidente que lo importante no es el poder de partida sino la capacidad de alianzas y que los grandes agentes pueden, si no consiguen apoyos adecuados, verse excluidos y expulsados, temporalmente al menos, del control de las decisiones.

2. En contraposición clara al axioma anterior, los medios y la comunicación están obligados a acomodarse a la diversidad de las sociedades.

Los ámbitos en que el poder diluido se juega no están definidos ni horizontal ni verticalmente. Al contrario, son inestables, se mueven casi en cada ocasión y sólo son previsibles por cálculo estadístico, por 
probabilidad sobre experiencias anteriores. Los modelos, en consecuencia, no sirven de mucho.

Los medios de comunicación, ante esa realidad, han optado por operar en diferentes planos, muy en línea con los modos de hacer del resto de las grandes industrias. En un plano dominante, más próximo, de mercado, han intentado segmentar las audiencias, especializar (tematizar se dice ahora) las informaciones y los programas, diversificar los contenidos por tiempos y espacios, poner a la vista una «oferta mosaico». Hay un plano menor, de carácter interior, en cuyo espacio los diferentes grupos de interés que configuran el medio o grupo pugnan por imponer su «libro de estilo» y que paraliza la evolución, tiende siempre al conservadurismo. Todas las organizaciones, por supervivencia de sus grupos dominantes, vuelven la vista hacia atrás, son esencialmente conservadoras. $\mathrm{Y}$ hay un tercer plano que es el comparativo, casi aristotélico: uno se mueve y define por sus contrarios y las decisiones estratégicas fundamentales se hacen operativas siempre desde la perspectiva del «benchmarking».

3. Los medios de comunicación son el referente de intelección de un sistema o de un proceso. No sólo son agentes sino también fuentes de análisis e intelección para el resto de los agentes.

Los dos poderes sociales clásicos (política y economía) se vieron obligados, a raíz del salto al postmodernismo, a establecer relaciones directas con sus usuarios y votantes. Para conseguir, en un ámbito muy competitivo, posiciones dominantes en el mercado han tenido que ir evolucionando, a lo largo de todo el siglo, hacia el uso diario y perpetuo de los medios de comunicación como instrumentos de acción directa y de relación inmediata entre ellos y quienes les producen el retorno de su supervivencia: los votantes y los compradores o usuarios. Esta actividad fue dando poco a poco a los medios un poder que saltó desde el ámbito de la intermediación al ámbito de posición dominante. No sólo por beneficios sino por la relación de dependencia creada, los medios y el sector industrial afín se sitúan como un sector indispensable.

La situación se consolida cuando el resto de los agentes sociales y económicos activos se fueron dando cuenta de que, con una estrategia adecuada, eran capaces de influir sobre la toma de decisiones fundamentales, a partir de su capacidad de estar presentes y de hacer llamadas de referencia en los medios de comunicación. Cuando hasta los niños conocen que para conseguir un objetivo les basta con amenazar 
el que cuentan algo a los periódicos o a la televisión, es evidente que las «cuerdas» en torno a las cuales se estructura una sociedad atraviesan y quedan reflejadas en los medios de comunicación

4. Los medios de comunicación permiten juzgar las decisiones y capacidades de los agentes sociales e históricos por sus resultados.

A cualquier responsable de decisiones en el ámbito privado se le termina juzgando por sus resultados. No en un único ámbito, no sólo resultados económicos, o incremento de las acciones, o beneficios sino también en posición en el mercado, conocimiento de marca, premios sociales, reputación, fidelización de empleados y clientes, etc.

Los periódicos son un excelente instrumento para llevar a cabo este mismo tipo de juicios en los personajes públicos, incluidos los propios agentes del sector de la información. Un político, desde principios al menos de este siglo, ha ido dejando sobre los medios de comunicación cuáles fueron su "plan de negocio« («business plan») o plan de ciclo electoral y su "plan anual» («marketing plan») o plan de ciclo corto y en los mismos medios de comunicación ha dejado fe del cumplimiento de sus objetivos a medio y corto. Este enfoque permite un análisis por objetivos y una revisión de la actividad pública a partir de sus consecuencias durante años.

¿Cuál es la conclusión? ¿Qué formatos metodológicos nuevos nos van a permitir una reinterpretación de la historia del periodismo que es lo mismo que decir de la historia española actual?. En un encuentro reciente de la Asociación de Historiadores de la Comunicación en la Universidad de Málaga se debatió largamente la cuestión. Se planteó allí el desarrollo metodológico previsible en la misma línea que aquí estamos siguiendo. Hacen falta análisis comparados e investigaciones de la sociedad española reciente que tengan como eje la comunicación con sus funciones y capacidades y hacen falta análisis del pasado desde sus repercusiones y perspectivas de futuro.

\section{Algunas propuestas para una relectura de los diarios madrileños después del 98}

En consideración lógica con las premisas apuntadas, existen toda una serie de cuestiones mal resueltas, en mi opinión, relacionadas con la interpretación histórica de principios de siglo y que deben ser 
revisadas desde la metodología para la Historia de la Comunicación que aquí proponemos. A modo de ejemplo proponemos, al menos, las que siguen.

1. La cuestión de la tirada de los diarios y su control por el timbre (Timoteo,81). Durante más de una década los investigadores han criticado y valorado negativamente los datos de las diferentes estadísticas oficiales, comparados con datos similares de otros países. Ese enfoque analítico, en la perspectiva que aquí desarrollamos, es totalmente equivocado. En primer lugar porque no se comparan datos homólogos sino solo cifras de tirada: con poblaciones distintas (la población de Madrid con la de Nueva York, París o Londres), mercados distintos (los diarios de Nueva York específicamente se distribuyen en la propia metrópoli mientras los de Madrid se distribuyen por toda la Península), situaciones sociales distintas. Específicamente son dignas de tener en cuentas las costumbres referidas a los hábitos de lectura: en Inglaterra, por ejemplo, un número vendido puede equivaler a uno o dos lectores; en España un número comprado puede tener detrás un indeterminado número de lectores. Son conocidos los ejemplos que Díaz del Moral propone en su Historia de las agitaciones campesinas andaluzas en los que, con frenesí, un artículo de Tierra y Libertad llegaba a docenas y docenas de hogares. Por eso, al igual que en la actualidad el EGM valora no sólo venta sino también número medio de lectores por ejemplar y que la medición oficial en radio y televisión valoran para la publicidad los GRPs, habría, para tener una situación aproximada de la influencia de los diarios a principios de siglo, que calcular para entonces esos mismos GRPs. Es simplemente falso ese aserto tan comunmente aceptado de que en España el número de lectores de prensa diaria por cada cien habitantes sea mucho menor que en Bélgica, Holanda o Inglaterra. La verdad es que aquí nadie lo ha calculado nunca dando por buenas cifras estadísticas de venta controladas por el timbre o por la OJD, pero que, como cualquier español que haya desayunado en cualquier bar sabe, no tienen nada que ver con el número real de lectores

2. La extraordinariamente buena imagen del regeneracionismo y de las generaciones de intelectuales del 98 y del 14 en España. Una cuestión tiene que ver con la calidad literaria de sus componentes en el caso de los literatos o con la estética de sus pronunciamientos y discursos en el caso de los regeneracionistas y otra distinta es la validez de sus planteamientos demostrada con el tiempo. Ellos fueron importantísimos gestores de una evolución social que terminó en una terrible catástrofe y no puede entenderse entonces como han conseguido man- 
tener tanta admiración y respeto. Desde la perspectiva que aquí proponemos, todos los «redentores» de principios de siglo vivieron una experiencia bastante patética. Estaban imbuidos, por una parte, de un poderoso elan ilustrado, con conciencia de que habían venido a este mundo para ser redentores de todos lo que no pensaban más o menos como ellos. Asentaban sus asertos, por otra parte, sobre un pensamiento binario y nada complejo, para nada acorde con una sociedad multifacética como era la occidental del siglo XX. Los techos de su intepretación social no pasaban de dividir a los españoles en buenos (los míos) y malos (los otros) o salvadores (los míos) y masas (los otros). Todo era miedos: a la desaparición de la fe en la tierra de la Virgen, a la rebelión de la masas, a golpes militares, a los curas, a los anarquistas, unos a otros. $\mathrm{Y}$ en tercer lugar, hasta es posible que nada es lo que parece y que la famosa Generación del 98 no pasó de ser una brillante operación de marketing literario de Ortega Munilla en Los Lunes de El Imparcial, o que detrás de las reformas educativas se ocultaba un indisimulado proselitismo de cuotas, votos, influencias y miedos.

3. Si, como es evidente, se producen en España movilizaciones de grupos organizados de masas desde muy a principios de siglo, en la semana trágica, en las huelgas parciales y generales, en la llegada de los socialistas al Congreso, por ejemplo y si, según nuestra hipótesis arriba justificada, esas movilizaciones no son posibles sin instrumentos o medios de comunicación de masas: ¿cómo se desarrolló aquí esa capacidad? Se puede seguir la historiografía convencional que interpreta todo el siglo XX español sin recurrir prácticamente nunca a los medios de comunicación, pero consideramos ese formato simplemente equivocado: el siglo XX no se puede entender, tampoco en España, sin los medios de comunicación y especialmente, sin los medios de comunicación te masas.

4. El otro factor que define este último siglo - también se indica $\mathrm{n}$ anterioridad en este artículo- es el hábito del consumo masivo bienes y servicios. En España ese hábito se va asentando como en el resto de occidente con el cambio de centuria y - no importa mucho diez años antes o diez años después- España es una sociedad de consumo de masas a mediados de siglo. Eso no pudo llegar a realizarse sin la intervención de medios de masas: ¿dónde están? ¿cómo se hizo posible ese desarrollo de hábitos y actitudes sin periódicos de tiradas millonarias?

Parece que, al menos como hipótesis, será necesario revisar conceptos e interpretaciones que son muy comunes en la historiografía de los 
últimos años y que de modo cómodo se han dejado llevar por propuestas de sistemas dados por buenos tal vez desde la leyenda negra o por formatos metodológicos demasiado lineales — toda la metodología marxista- o útiles para otras realidades - Annales - pero no sé si para las nuestras.

\section{Bibliografía citada}

AlCAIDE, J., 2000: «La renta nacional de España y su distribución. Serie años 1898 a 1998" en Velarde Fuertes,J., 1900-2000. Historia de un esfuerzo colectivo. Fundación BSCH-Planeta, Madrid.

Fuentes/Fdz Sebastían, 1997: Historia del Periodismo Español, Sintesis, Madrid.

Gómez Mompart, J., 1989: «¿Existió en España prensa de masas? La prensa en torno a 1900 ", en Timoteo (1989).

Gómez Mompart, J., (ed.), 1969: Metodologías para la Historia de la Comunicación Social, Univ. Autónoma, Bellaterra, 1996.

MAINER, J., 1975: La edad de plata (1902-1931), Barcelona.

MoReno SARDÁ, A., 1998: La mirada informátiva, Bosch, Barcelona.

Salaún/Serrano, 1900 en España, Espasa-Calpe, Madrid, 1991.

Sánchez Aranda/Barrera, 1992: Historia del Periodismo Español: desde sus orígenes hasta 1975, Eunsa, Pamplona.

SECo, C., 1993: “Las ideologías políticas» en Historia de España XXXIX, Espasa Calpe. Madrid.

Timoteo Alvarez, J., 1981: Restauración y Prensa de Masas: los engranajes de un sistema, Eunsa, Pamplona.

Timoteo Alvarez, J., 1987: Historia y Modelos de Comunicación en el siglo XX, Ariel, Barcelona.

Timoteo Alvarez, J. (editor), 1989: Historia de los Medios de Comunicación en España: Periodismo, Imagen y Publicidad (1900-1990), Ariel, Barcelona. 